\title{
Perfil fitoquímico e determinação da atividade antimicrobiana de Syzygium cumini (L.) Skeels (Myrtaceae) frente a microrganismos bucais
}

CARTAXO-FURTADO, N.A.D.E.O. ${ }^{*}$; SAMPAIO, T.O.'; XAVIER, M.A.'; MEDEIROS, A.D.D.E.'; PEREIRA, J.V. ${ }^{1}$ 1Universidade Estadual da Paraíba, Rua Baraúnas, 351, Bodocongó, Campina Grande, Paraíba. CEP: 58429500, Brasil. *Autor para correspondência: nathalia_cartaxo@hotmail.com

RESUMO: Este estudo teve como objetivo determinar o perfil fitoquímico e avaliar a atividade antimicrobiana in vitro do extrato etanólico da casca do caule de Syzygium cumini(L.) Skeels frente a microrganismos bucais. O perfil fitoquímico do extrato foi traçado através da determinação espectrofotométrica quantitativa para verificar o teor de taninos, flavonóides, saponinas e polifenóis. A atividade antimicrobiana foi determinada através da Concentração Inibitória Mínima (CIM), por meio da técnica de microdiluição em caldo, utilizando-se as seguintes linhagens de microrganismos: Streptococcus mutans (25175), Streptococcus oralis (10557) e Candida albicans (10231). Uma quantidade apreciável de fitocontituintes foi observada, especialmente de taninos $(100,58 \pm 1,81)$. Os extratos apresentaram atividade antimicrobiana inibindo o crescimento das linhagens em estudo, destacando-se essa atividade sobre 0 crescimento de $C$. albicans $(\mathrm{CIM}=250 \mu \mathrm{g} / \mathrm{mL})$. Já as CIMs para Streptococcus foram baixas. Diante dos resultados expostos, pode-se concluir que o perfil fitoquímico foi traçado e que, dentre os microrganismos testados, o extrato etanólico da casca de S. cumini apresentou forte potencial de inibição sobre o crescimento de $C$. albicans e fraca inibição frente aos Streptococcus testados. Este estudo sugere que mais pesquisas devem ser realizadas dando continuidade à bioprospecção, por meio de análises experimentais com essa espécie vegetal, objetivando, no futuro, que essa planta possa ser utilizada clinicamente para tratar candidose bucal.

Palavras-chave: Syzygium cumini, Fitoquímica, Streptococcus, Candida albicans.

\begin{abstract}
Phytochemical profile and determination of antimicrobial activity of Syzygium cumini (L.) Skeels (Myrtaceae) against oral microorganisms. This study aimed to determine the phytochemical profile and to evaluate the in vitro antimicrobial activity of the ethanol extract of stem bark of Syzygium cumini (L.) Skeels against oral microorganisms. The phytochemical profile of the extract was traced through a quantitative spectrophotometric determination in order to check the tannin, flavonoids, saponins, and polyphenols content. The antimicrobial activity was determined through minimum inhibitory concentration (MIC) by the broth microdilution technique, using the following strains of microorganisms: Streptococcus mutans (25175), Streptococcus oralis (10557) and Candida albicans (10231). An appreciable amount of fitocontituintes was observed, particularly the tannin $(100.58 \pm 1.81)$. The extracts showed antimicrobial activity, inhibiting the growth of the strains under study, with this activity being more intense on the growth of $C$. albicans ( $\mathrm{MIC}=250 \mathrm{mg} / \mathrm{mL}$ ). On the other hand, the MICs of the Streptococcus were low. In face of the mentioned results, we can conclude that the phytochemical profile was traced and that, among the tested microorganisms, the ethanol extract of $\mathrm{S}$. cumini bark showed strong potential to inhibit the growth of $C$. albicans and weak inhibition against the Streptococcus tested. This study suggests that more research should be done by proceeding with the bioprospecting, through experimental tests with this plant's species, aiming that in the future this substance can be used clinically for the treatment of oral candidiasis.
\end{abstract}

Keywords: Syzygium cumini, Phytochemistry, Streptococcus, Candida albicans 


\section{INTRODUÇÃO}

Os microrganismos da microbiota bucal podem viver harmonicamente na cavidade bucal ou, quando há desequilíbrio na homeostase, podem provocar doenças no indivíduo, tais como cárie, doença periodontal e candidose bucal (Mukherjee et al., 2014). Cárie dentária e doença periodontal são patologias comuns na população em geral e em várias regiões do mundo, sendo a cárie considerada um problema de saúde pública, provocada por bactérias do gênero Streptococcus (Allaker, 2010; Olabisi et al. 2015) e outros fatores relacionados ao hospedeiro. A candidose bucal é uma infecção oportunista que tem como principais sintomas a dor e/ou queimação e tem por agente etiológico leveduras do gênero Candida, sendo a Candida albicans a espécie mais prevalente (Samson, 2013).

Matérias primas derivadas de plantas medicinais podem ser uma alternativa para serem incorporadas às novas formulações farmacêuticas para o tratamento de doenças, pois os produtos naturais têm a capacidade de causar menos efeitos colaterais do que os medicamentos sintéticos. Além disso, apresentam várias propriedades terapêuticas devido à presença de metabólitos secundários, podendo ser aplicada também para ajudar a remover o biofilme dental e evitar que afecções bucais se instalem (Jesus et al., 2010; Nwodo et al., 2011).

Atualmente, as pesquisas com produtos naturais têm sido desenvolvidas de maneira satisfatória na odontologia, pelo fato de se buscar novos com menor toxicidade e maior atividade farmacológica, além de ter um bom custo e benefício para a população. O Brasil apresenta um grande potencial para o desenvolvimento de novos medicamentos a partir de plantas medicinais, pois possui $25 \%$ da flora mundial (Francisco, 2010).

Syzygium cumini(L.) Skell é uma planta que pertence à família Myrtaceae, originária da Índia e naturalizada no Brasil. As folhas são ricas especialmente em taninos e saponinas (Loguércio et al., 2005; Migliato, 2005; Swami et al. (2012) afirmam que as folhas contêm ácido gálico, metilgalato, canferol, miricetina, ácido elágico, ácido clorogênico, quercetina, nilocitina, $\beta$-sitosterol, ácido betulínico. A casca do caule é rica em ácido betuliníco, friedelina, epifriedelanol, $\beta$-sitosterol, eugenina, éster de ácido graxo, quercetina, campferol, miricetina, ácido gálico e ácido elágico, bergeninas, flavonóides e taninos (Ayyanar \& Subash-Babu, 2012).

As diferentes partes dessa planta, popularmente conhecida como jambolão, são amplamente utilizadas na medicina popular. $O$ caule e a casca do fruto possuem propriedade hipoglicemiante para controle de diabetes, atividade anticarcinogênica, antimicrobiana e antiinflamatória.
Os frutos também têm ação hipoglicemiante e antioxidante, destaca-se a presença do ácido elágico que confere características antioxidantes e anticarcinogênicas (Lorenzi \& Matos, 2002; Vizzoto \&Fetter, 2009). Costa et al. (2009) revela que a decocção da casca do caule de S. cumini é utilizada na forma de bochechos para tratar ulcerações aftosas, estomatites, infecções da garganta e outras doenças da cavidade bucal.

Diante do exposto, nota-se a importância de se pesquisar novas substâncias, preferencialmente de origem natural que tenha uso popular descrito, o que favorece sua utilização também por grupos populacionais com acesso restrito à assistência em saúde. Por isso, essa pesquisa visa determinar 0 perfil fitoquímico e avaliar a atividade antimicrobiana in vitro do extrato etanólico da casca do caule de Syzygium cumini(L.) Skeels frente a microrganismos bucais.

\section{MATERIAL E MÉTODOS \\ Obtenção e preparação do material vegetal}

As cascas do caule foram obtidas a partir de árvores de jambolão (Syzygium cumini (L.) Skeels Myrtaceae), cultivadas na chácara Flor do Campo, localizada na zona rural do Município de Campina Grande (CG), Paraíba, Brasil. Preparou-se uma exsicata, a qual foi depositada no Herbário Arruda Câmara, da Universidade Estadual da Paraíba (UEPB) sob voucher 084 e identificada por um botânico da instituição. As mesmas foram coletadas em novembro de 2013. O material vegetal foi seco/ desidratado em estufa de secagem com circulação de ar (FANEM - Modelo 330/5), a temperatura de $45^{\circ} \mathrm{C}$ até a estabilização final do peso e processados em moinho de facas (Solab Científica) de 10 mesh, para se obter granulometria padronizada, conforme preconiza a Brasil (2010).

\section{Preparação do extrato vegetal}

Em seguida, foi preparado o extrato etanólico pelo esgotamento da droga vegetal por meio do processo de percolação com renovação de solvente a cada cinco dias; utilizou-se a proporção de $100 \mathrm{~g}$ da droga vegetal moída para $1000 \mathrm{~mL}$ de etanol (Ouedraogo et al., 2005). O extrato foi utilizado para os ensaios fitoquímicos e determinação da atividade antimicrobiana.

\section{Testes fitoquímicos}

O perfil fitoquímico do extrato foi traçado por meio de quantificação espectroscópica, em triplicata, e analisado estatisticamente quanto ao desvio padrão sendo esse realizado de acordo com os seguintes métodos: 


\section{Determinação do teor de taninos condensados}

A determinação do conteúdo de taninos condensados seguiu o método descrito por Makkar \& Becker (1993). A 0,5 mL da amostra do extrato vegetal foi adicionado $3 \mathrm{~mL}$ de uma solução de vanilina (4\% p/v em metanol); em seguida, adicionouse $1,5 \mathrm{~mL}$ de ácido clorídrico $(\mathrm{HCl})$ concentrado (37\%). A reação ocorreu em tubos de ensaio, mergulhados em água a cerca de $22^{\circ} \mathrm{C}$. A leitura foi feita a $500 \mathrm{~nm}$, contra um branco composto pela solução de vanilina, $\mathrm{HCl}$ e uma solução de etanol $50 \%$ (v/v) em água. A curva de calibração foi obtida a partir de uma solução padrão de catequina obtida pela dissolução de $10 \mathrm{mg}$ do padrão em $100 \mathrm{~mL}$ de metanol. A partir dessa solução foram realizadas diluições em triplicata, de forma a obter soluções de catequina nas concentrações de 10, 20, 30, 40, $50,60,70,80,90$ e $100 \mu \mathrm{g} / \mathrm{mL}$. A concentração de taninos condensados foi expressa em miligramas equivalentes de catequina.

\section{Determinação do teor de flavonóides totais}

Meda et al. (2005) sugere um método para a quantificação de flavonóides totais, o qual foi aplicado nesta pesquisa. Obteve-se uma mistura de $5 \mathrm{~mL}$ de extrato dissolvido em metanol no mesmo volume de uma solução (em metanol) de Cloreto de Alumínio $\left(\mathrm{AlCl}_{3}\right)$ a $2 \%(\mathrm{p} / \mathrm{v})$. Após 10 minutos de repouso foi realizada a leitura da absorbância a 415 $\mathrm{nm}$, contra um branco composto pela solução de $\mathrm{AlCl}_{3}$. O teor de flavonóides totais foi determinado pela curva de calibração utilizando quercetina (Sigma-Aldrich) como padrão nas concentrações de $2,4,6,8,10,13,16,19,22,26,28$ e $30 \mu_{g m L}-1$ e expresso em mg equivalente de quercetina.

\section{Determinação do teor de polifenóis totais}

A determinação do teor de polifenóis totais seguiu a metodologia proposta por Chandra \& Mejía (2004), com adaptações, através de espectroscopia na região do visível por meio do método de FolinCiocalteu. Submeteu ao estado de repouso por dois minutos, uma mistura de $1 \mathrm{~mL}$ da solução aquosa do extrato com $1 \mathrm{~mL}$ do reagente de Folin-Ciocalteau $1 \mathrm{~N}$. Em seguida, $2 \mathrm{~mL}$ de uma solução aquosa de $\mathrm{Na}_{2} \mathrm{CO}_{3}$ a $20 \%$ (p/v) foi adicionada, e a mistura permaneceu em repouso por mais 10 minutos. Por último, foi feita a leitura da absorbância a $757 \mathrm{~nm}$ em espectrofotômetro (Shimadzu® UV mini - 1240), contra um branco composto por água destilada, reagente de Folin-Ciocalteau e solução a $20 \%$ de $\mathrm{Na}_{2} \mathrm{CO}_{3}$. A curva de calibração foi obtida a partir de soluções de ácido gálico nas concentrações de $1,3,6,9,12,15,20,25,30,35$, e $40 \mu \mathrm{g} / \mathrm{mL}$. A concentração de polifenóis foi expressa em miligramas equivalentes de ácido gálico.
Determinação da atividade antimicrobiana

Os testes de atividade antimicrobiana foram realizados através da técnica de microdiluição em caldo descrita no documento Clinical and Laboratory Standards Institute (CLSI, 2005; CLSI, 2002), com adaptações. Foram selecionadas cepas padrão American Type Culture Collection (ATCC): Streptococcus mutans (25175), Streptococcus oralis (10557) e Candida albicans (10231). Após serem feitos testes prévios com a concentração do extrato variando de $2 \mathrm{mg} / \mathrm{mL}$ até $8 \mathrm{mg} / \mathrm{mL}$ frente às cepas escolhidas, a melhor concentração do extrato selecionada para prosseguir a determinação da Concentração Inibitória Mínima foi a de $4 \mathrm{mg} / \mathrm{ml}$ (Mello-Peixoto et al., 2014).

\section{Preparo dos inóculos}

O preparo dos inóculos para os testes de suscetibilidade foram realizados seguindo as recomendações do protocolo M7-A6 para bactérias (CLSI, 2005) e M27-A2 para leveduras (CLSI, 2002). Culturas de bactérias incubadas por 24 horas em gerador de anaerobiose (Probac) foram preparadas e adicionadas em solução salina $(0,85 \%)$ esterilizada $(5 \mathrm{~mL})$. Sua absorbância foi ajustada entre 0,08 a 0,10 a um comprimento de onda de $625 \mathrm{~nm}$ em fotocolorímetro Bioespectro, modelo SP-22, originando uma concentração equivalente a $1,5 \times 10^{8}$ UFC. A partir disso, foi realizada uma diluição seriada obtendo-se ao final da mesma uma concentração de $1 \times 10^{6}$ UFC. Os testes de suscetibilidade para Candida albicans foram realizados através do método de microdiluição em caldo seguindo as recomendações do protocolo M27-A2 para leveduras (CLSI, 2002). A concentração do inóculo, após ajuste em fotocolorímetro $(530 \mathrm{~nm})$, correspondeu a $5 \times 10^{\circ} \mathrm{cé} / \mathrm{s} / \mathrm{mL}$ e, após diluições sucessiva a 2,5 $\times 10^{3}$ céls $/ \mathrm{mL}$.

\section{Determinação da concentração inibitória mínima (CIM)}

Foram distribuídos nos orifícios de cada coluna, $100 \mu \mathrm{L}$ do caldo BHI e Sabouraud para bactéria e fungo, respectivamente. Na coluna 1 linha A foram acrescentados $50 \mu \mathrm{L}$ (micropipeta Eppendorf, Researchplus, 20-200 $\mu \mathrm{L}$ ) de solução da amostra testada, de concentração conhecida, sendo estes referentes ao controle de esterilidade das amostras. Em seguida, na linha B foram adicionadas $100 \mu \mathrm{L}$ dos extratos para em seguida efetuar uma diluição seriada nos poços consecutivos, retirandose $100 \mu \mathrm{L}$ do poço de maior concentração para o poço seguinte. Os $100 \mu \mathrm{L}$ finais foram desprezados.

A partir das suspensões padrões de cada espécie de microrganismo foram pipetados $100 \mu \mathrm{L}$ dessa suspensão em cada poço. As primeiras colunas correspondem ao controle positivo (gluconato de

Rev. Bras. PI. Med., Campinas, v.17, n.4, supl. III, p.1091-1096, 2015. 
clorexidina $0,12 \%$ para bactéria e nistatina 100.000 $\mathrm{UI} / \mathrm{mL}$ para a levedura) e ao controle do solvente (etanol $40 \%$ ) para verificar se o etanol seria capaz de inibir o crescimento das células viáveis. As placas foram incubadas por $24-48$ horas a cerca de $36^{\circ} \mathrm{C}$ em atmosfera aeróbia, microaerofilia ou anaeróbia dependendo da exigência do microrganismo (CLSI, 2002; CLSI, 2005).

Para a leitura dos resultados da CIM, foram adicionados $20 \mu \mathrm{L}$ da solução de cloreto de resazurina 0,01\% (Sigma AldrichChemistry ${ }^{\circledR}$ ). As placas foram re-incubadas por um período de 40 minutos para a levedura, e por duas horas para bactérias. A CIM foi definida como a menor concentração da amostra, capaz de impedir o aparecimento de coloração rósea, conferida ao meio quando as células apresentam atividade respiratória (CLSI, 2005).

\section{RESULTADOS E DISCUSSÃO Perfil fitoquímico}

Neste estudo, o composto fitoquímico que apresentou maior concentração no extrato etanólico de casca de $S$. cumini foram taninos, seguido de polifenóis e flavonóides (Tabela 1).

Quando o etanol é utilizado como solvente, devido à sua polaridade, pode extrair do material vegetal as seguintes classes químicas: taninos, saponinas, alcalóides, flavonóides, triterpenóides e heterosídeos em geral. Sendo assim, os resultados do perfil fitoquímico mostram que os mesmos foram coerentes em relação ao tipo de extrato escolhido, ou seja, o extrato etanólico (Simões et al., 2007).

Os resultados encontrados corroboram com o estudo de Djipa et al. (2000) que ao analisarem o extrato acetônico da casca da árvore de S. cumini por reações cromatográficas e colorimétricas, os taninos foram os principais compostos que prevaleceram e, inclusive, foram identificados ambos os taninos hidrolisáveis e condensados. Jayachandra \& Devi (2012) investigaram a composição química em extrato metanólico de caule de S. cumini e verificaram a presença de flavonóides, fenóis, saponinas, taninos, esteróis e terpenóides. Análise fitoquímica qualitativa realizada por Kuncha et al. (2012) mostrou a presença de fenóis, terpenos, taninos, saponinas, fitoesteróis, carboidratos, flavonóides e ácidos aminados em extratos metanólicos e aquosos na casca do caule de S. cumini. Vale ressaltar, no atual estudo, a importância de se ter quantificado no extrato de casca de jambolão, a presença marcante de taninos, suas atividades biológicas são extremamente promissoras. Estudos apontam sua ação antimicrobiana, reparação de tecidos e regularização de proteínas e enzimas, grupos de taninos estão sendo estudados minuciosamente, tendo por finalidade encontrar uma droga efetiva contra o vírus HIV (Brunet et al., 2003). Além disso, taninos constituem uma das classes de maior destaque devido a sua ampla distribuição no reino vegetal e suas importantes atividades biológicas, dentre elas antibacteriana e antifúngica (Diniz, 2006; Kaiser et al., 2010).

A quantidade de polifenóis foi menos expressiva quando comparado com estudo de Kuskoski et al. (2006) que encontrou um teor de $229,6 \mathrm{mg}$ de polifenóis total na polpa do fruto do Jambolão.

Segundo Castro et al. ( 2012) os polifenois são altamente benéficos à saúde, dentre seus efeitos biológicos são válidos citar a inibição da proliferação celular, potencial antialérgico, atividade antimicrobiana, como também sua atividade antiinflamatória. A atividade antimicrobiana dos compostos fenólicos é devida sua ação na parede celular dos microrganismos combinando-se assim com as suas adesinas, de forma a comprometer a adesão do microrganismo sobre a superfície celular.

De maneira geral, os resultados do perfil fitoquímico sugerem potencialidade para atividade antimicrobiana devido aos constituintes fitoquímicos identificados e quantificados satisfatoriamente como taninos, polifenóis e de flavonóides que podem estar associados com atividade antimicrobiana (Souza et al., 2013).

\section{Atividade antimicrobiana}

Diante dos resultados do perfil fitoquímico e da utilização na medicina popular da casca de S. cumini como anti-séptico, adstringente em ulcerações bucais, gengivite e estomatite (Migliato et al., 2006; Costa et al., 2009) pode-se observar nesse estudo que $S$. cumini tem a capacidade de inibir o crescimento de Candida albicans, porém os resultados para Streptococcus não foram satisfatórios, visto que o extrato apresentou inibição fraca diante do crescimento celular dessas bactérias (Tabela 2).

Aligiannis et al. (2001) sugerem uma classificação de Concentração Inibitória Mínima: CIM até $500 \mu \mathrm{g} / \mathrm{mL}$ são inibidores potentes; CIM

TABELA 1. Composição química quantitativa do extrato etanólico de casca de $S$. cumini L. Skeel.

\begin{tabular}{cccc}
\hline Composto & Polifenóis & Flavonóides & Taninos \\
\hline Teor $(\mathrm{mg} / \mathrm{g})$ & $41,84 \pm 3,24$ & $1,98 \pm 0,39$ & $100,58 \pm 1,81$
\end{tabular}

\footnotetext{
${ }^{*} \mathrm{mg}$ de equivalentes do padrão de referência por $\mathrm{g}$ de extrato.
} 
TABELA 2. Determinação da Concentração Inibitória Mínima (CIM) do extrato etanólico de casca de S. cumini

\begin{tabular}{lc}
\hline Microrganismos & CIM $(\mu \mathrm{g} / \mathrm{mL})$ \\
\hline S. mutans (25175) & $>1600$ \\
S. oralis (10557) & $>1600$ \\
C. albicans (10231) & 250 \\
\hline
\end{tabular}

entre 600 e $1500 \mu \mathrm{g} / \mathrm{mL}$ são inibidores moderados; CIM acima de $1600 \mu \mathrm{g} / \mathrm{mL}$ são inibidores fracos. Por isso, pode-se considerar que frente à $C$. albicans, jambolão apresentou-se como um forte inibidor de crescimento, pois o valor da CIM foi igual a $250 \mu \mathrm{g} /$ $\mathrm{mL}$.

Nota-se na literatura que as pesquisas com Syzygium cumini têm sido feitas geralmente frente às bactérias intestinais e à Candida spp. Pouco é descrito sobre a sensibilidade de bactérias da cavidade oral envolvidas na formação do biofilme dental.

Apesar de estar descrito na literatura (Migliato et al., 2006; Costa et al., 2009) sobre o uso popular em forma de bochechos, pouco é estudado sobre a atividade antimicrobiana de extrato etanólico da casca do caule de Syzygium cumini, pela técnica de microdiluição; até mesmo pela técnica de disco-difusão em Ágar encontram-se limitações nos estudos com microrganismos da cavidade oral. A ênfase é maior para as folhas e frutos. Por exemplo, em pesquisa desenvolvida por Oliveira et al. (2007) foi avaliada a atividade antibacteriana do extrato hidroalcoólico de folhas de jambolão sobre doze isolados bacterianos, o jambolão inibiu de maneira mais expressiva o crescimento de Candida krusei, apresentando também atividade significativa contra as cepas multirresistentes e padrão de Pseudomonas aeruginosa, Candida albicans e Neisseria gonorrhoeae. Assim como na atual pesquisa, em estudo realizado por Oliveira et al. (2007), jambolão mostrou forte inibição sobre o crescimento de fungos do gênero Candida.

Neste sentido, os resultados obtidos mostram ser de grande valia a realização de pesquisas in vivo, além de testes de toxicidade para que desta forma, futuramente, os extratos de S. cumini possam ser utilizados, clinicamente, no tratamento da candidose oral visto que, os estudos preliminares desta pesquisa mostram que o extrato possui metabólitos secundários o que lhes confere forte inibição sobre Candida albicans do extrato de casca do caule de jambolão e fraca atividade frente aos Streptococcus. Portanto, este estudo sugere que outras pesquisas sejam realizadas dando continuidade à bioprospecção, por meio de análises experimentais com essa espécie vegetal.

\section{REFERÊNCIAS}

ALLAKER, R. P. The use of nanoparticles to control oral biofilm formation. Journal of Dental Research, v. 89, n. 11, p. 1175-1186, 2010.

ALIGIANNIS, N, et al., Composition and antimicrobial activity of the essential oils of two Origanum species. Journal of Agricultural and Food Chemistry, v. 40, p. 4168-4170, 2001.

AYYANAR, M.; SUBASH-BABU, P. Syzygium cumini (L.) Skeels: A review of its phytochemical constituents and traditional uses. Asian Pacific Journalof Tropical Biomedicine, v. 2, n. 3, p. 240-246, 2012.

BRUNET, J. R et al., Inhibición de lareplicacióndelvirus de inmunodeficiencia humana por extractos de taninos de Pinus caribaeaMorelet, Revista Cubana de farmácia, v.37 n.2, 2003.

CASTRO, L. C. de, et al., Avaliação da atividade antimicrobiana de extrato aquoso e etanólico de Acanthos permumaustrale. Caderno pedagógicov. 9, n. 2, p. 153-161, 2012.

CHANDRA, S.; MEJÍA, E. G. Polyphenolic Compounds, Antioxidant Capacity and Quinone reductase activity of an aqueous extract of Ardisiacompressa in comparison to mate (Ilexparaguariensis) and green (Camellia sinensis) Teas. Journal of Agricultural and Food Chemistry, v. 52, n.11, p. 3583-3589, 2004.

CLSI.National Committee for Clinical Laboratory Standards.Norma M27-A2. Método de referência para testes de diluição em caldo para determinação da sensibilidade de leveduras à terapia antifúngica.2.ed. Pennsylvania: NCCLS; 2002. 51p.

CLSI.National Committee for Clinical Laboratory Standards.Norma M7-A6. Metodologia dos testes de sensibilidade a agentes antimicrobianos por diluição para bactéria de crescimento aeróbico. 6.ed. Pennsylvania: NCCLS; 2005. 81 p.

COSTA, A. C. B. P. da; et al., Atividade antifúngica dos extratos glicólicos de Rosmarinus officinalis Linn e Syzygium cumini Linn. sobre cepas clínicas de Candida albicans, Candida glabrata e Candida tropicalis. Revista de Odontologia da UNESP, v. 38, n. 2, 111-116, 2009.

DINIZ, L. R. L. Efeito das saponinas triterpênicas isoladas de raízes da Ampelozizyphus amazonicus Ducke sobre a função renal. 2006. 116 f. Dissertação (Mestrado em Fisiologia e Farmacologia) - Universidade de Minas Gerais, Belo Horizonte

DJIPA, D.C., et al., Antimicrobial activity of bark extracts of Syzygium Jambos (L.) Alston (Myrtaceae), Journal of Ethnopharmacology v. 71, p. 307- 317, 2000.

BRASIL. FARMACOPEIA BRASILEIRA. 5 ed. v. 1. Brasília: Agência Nacional de Vigilância Sanitária, 2010.

FRANCISCO, K.M.S. Fitoterapia: uma opção para o tratamento odontológico. Revista Saúde, v. 4, n.1, p.18-24, 2010.

JAYACHANDRA, K; DEVI, V. S. Invitroantioxidant activity of methanolic extract of Syzygiumcumini Linn.bark. Asian Journal of Biomedical and Pharmaceutical Sciences, v. 2, n. 12,p. 45-49, 2012.

Rev. Bras. PI. Med., Campinas, v.17, n.4, supl. III, p.1091-1096, 2015. 
JESUS, R. P. F. S. de, et al., Ação antibacteriana e antiaderente de Pithecellobium cochliocarpum (gomez) Macbr sobre microrganismos orais. Odontologia Clínico-Científica (Online),v.9, n.4, p.331-335, 2010.

KAISER, S. et al., Estudo da relação estrutura-atividade de saponinas hemolíticas e/ou imunoadjuvantes mediante uso de análise multivariada. Revista Brasileira de Farmacognosia, v.20, n.3, jul. 2010.

KUNCHA J, et al., . In vitro evaluation of nitric oxide scavenging activity of methanolic and aqueous extract of Syzygium cumini Linn. Bark (Myrtaceae). International Journal of Pharmaceutical Sciences Review, v. 3, n.2, p. 615-619, 2012.

KUSKOSKI, E. M. et al., Frutos tropicais silvestres e polpas de frutas congeladas: atividade antioxidante, polifenóis e antocianinas. Ciência Rural v. 36, n.4, p. 1283-1287, 2006.

LOGUÉRCIO, A. P.; et al., . Atividade antibacteriana de extrato hidroalcoólico de folhas de jambolão (Syzygiumcumini L.Skells, Ciência Rural, v. 35, n. 2, p. 371-376, 2005.

LORENZI, H. E.; MATOS, F.J. DE A. Plantas medicinais no Brasil/ Nativas e exóticas. Instituto Plantarum, Nova Odessa, v.1, n. 2, p.249-254, 2002.

MAKKAR, H. P. S.; BECKER, K. Vanillin-HCl method for condensed tannins: Effect of organic solvents used for extraction of tannins. Journal of Chemical Ecology, n. 4, v. 19,p.613-621, 1993.

MEDA, A., et al. Determination of the total phenolic, flavonoid and proline contents in Burkina Fasan honey, as well as their radical scavenging activity. FoodChemistry, v. 91, p. 571-577, 2005.

MELLO-PEIXOTO, E.C. T. de, et al., .Extrato hidroalcoólico de Punica granatum Linn. sobre Staphylococcus spp. isolado de leite bovino, Cadernos de Agroecologia, v. 9, n.1, 2014.

MIGLIATO, K. F. Syzygium cumini (L.) SkeelsJambolão: estudo farmacognóstico, otimização do processo extrativo, determinação da atividade antimicrobiana do extrato e avaliação da atividade anti-séptica de um sabonete líquido contendo o referido extrato. Dissertação [Mestrado]. Faculdade de Ciências Farmacêuticas de Araraquara, UNESP.

MIGLIATO, K. F., et al., Ação farmacológica de Syzygium cumini(L.) Skeels. Acta Farmaceutica Bonaerense, v.25, p.310-314, 2006.

MUKHERJEE, P. K; et al., Oral Mycobiome Analysis of HIV-Infected Patients: Identification of Pichia as an Antagonist of Opportunistic Fungi.PlosPathogens, v. 10, n.3, 2014.

NWODO, U. U. et al., Assessment of Tamarindus indica extracts for antibacterial activity. International Journal of Molecular Sciences, v. 12, p. 6385-6396, 2011.

OLABISI, A. A. et al., Prevalence of dental caries and oral hygiene status of a screened population in Port Harcourt, Rivers State, Nigeria. Journal of International Society of Preventive and Community, v. 5, n. 1, p. 59-63, 2015.

OLIVEIRA, G. F. et al., Antimicrobial activity of Syzygium cumini (Myrtaceae) leaves extract. Brazilian Journal of Microbiology, v.38, n.2, p.381-384, 2007.

OUEDRAOGO, S, et al., Pharmacological evaluations for the relaxant effect of the hydroalcoholic extract of Tapinanthus dodoneifolius on rat trachea. African Journal of Traditional, Complementary, and Alternative, v.2, p.166-176, 2005.

SAMSON N.G. Managing patients with oral candidiasis. Journal of the Canadian Dental Association, v. 79, n.122, 2013.

SIMÕES, C.M.O. Farmacognosia: da planta ao medicamento. Florianópolis: Editora da UFSC; 2007, 483 p.

SOUZA, R.K.D, et al., Aspectos etnobotânicos, fitoquímicos e farmacológicos de espécies de Rubiaceae no Brasil. Revista Cubana de Plantas Medicinais, v.18, n.1, p.140-156, 2013.

SWAMI, S. B, et al., Jamun (Syzygium cumini (L.)): A Review of its food and medicinal uses. Food and Nutrition Sciences, v. 3, p. 1100-1117, 2012.

VIZZOTO, M.; FETTER. R. M. Jambolão: o poderoso antioxidante. Embrapa - clima temperado; 2009. 